\title{
Study on the Current Situation, Problems and Solutions of College Student Network Anchors
}

\author{
Yongzhen Shen \\ Xi'an Peihua University, Xi’an, China
}

Keywords: College Students Network Anchor; Current Situation; Problems; Solutions

\begin{abstract}
In recent years, with the industry of college student anchors entering the public view as an emerging industry, the number of college student anchors in commercial applications shows a blowout development. This paper first gives brief description of the current situation of the development of college student anchors, discusses the concept of college student anchors, the contents and the current situation of the industry, and then elaborates the development plight that college student anchors market is can be said to be in chaos driven by profits; and finally puts forward an effectively interactive development strategy of the four parties, "government + capital + platform + anchor".

In recent years, the live streaming market, stimulated by huge profits, has attracted much market attention and a massive influx of capital. The number of college student network anchors, with the continuous development of live streaming platforms, also increases rapidly. With the advantage of the large number of Internet users, college student network anchors have created some economic value. The market of college student network anchors is hot, however, due to the low requirements of college student network anchors that live streaming can be achieved just with a phone and a selfie stick, the market of college student network anchors is a admixture of good and evil, even some anchors attract fans by any kind of means, resulting in the obvious "vulgarization" phenomena in general. We need to analyze in depth and take corresponding solutions about how to address the existing problems in the industry and seek a healthy development of the college student network anchors industry.
\end{abstract}

\section{General description of college student network anchors industry}

\subsection{College student network anchor}

The college student network anchors refer to those who plan, edit, record, make and interact with the audience through Internet platforms with computer, mobile phone, selfie sticks and so on. At present, the income of college student network anchors mainly comes from three parts: Firstly, fan reward. During the interaction with fans, college student network anchors will receive the virtual gifts from fans, one half of which is usually taken away by the platform, and the remaining half is allocated by the anchor and the team; Secondly, the platform salary. This part is from the platform to retain popular anchor after signing the contract with them; Thirdly, the allocated salary from cashing the advertising, e-commerce, activities hosted, and the flow. At present, college students are the main force of the live streaming platform. According to the statistics of author's research team, $31 \%$ of the media major students have the experience of network anchors. They think that the network anchor can not only exert their professional expertise, but also gain economic benefits and alleviate the pressure on parents.

\subsection{Main contents of college student network anchor}

(1) Game anchor: One type of this kind of college student network anchor is a game master. Such anchor is generally the game master, and what people see in the live streaming is stimulating masterstroke operations; The other is the funny type. These anchors bring fun to fans in a humorous language through online games or offline activities. 
(2) Chat and making friends: This type of college students is only for chatting and making friends to kill time; Or for purposeful relations. This type is more casual, and only conducted occasionally by some college students.

(3) Pan-entertainment anchor: This type is to provide wonderful performances for fans with live streaming as a medium, and fully demonstrate their value. Or to pursue economic interests by any means, like vulgar live streaming.

(4) 'Live streaming + anchor: There are more college student network anchors in the form of live streaming+E-commerce, live streaming+education, live streaming+sports, and live streaming+travelling. Live streaming+education has become the popular live streaming form among many college students form elite universities to help those with learning difficulties solve their leaning problems.

\subsection{Current situation of college student network anchors}

First of all, let's see the current situation of national network anchors: Live streaming shows a rapid development trend and network anchors emerge one after another. In the first year of live streaming, the number of domestic live streaming platforms reached 237, with the number of live streaming users exceeding 344 million, accounting for $47.1 \%$ of the total number of Internet users. There are nearly 200 domestic live streaming platforms with the total market exceeding 25 billion yuan. Show live streaming, the core of network anchor market all the time, has been sluggish in the year of 2017 though as the mainstream. Live streaming platforms begin the transformation one after another, with high-quality live streaming contents as the core, to seek wider and deeper innovation in contents of platforms. The proportion of male anchors in 2017 has reduced to $29.12 \%$ from 54\% of 2016, and female anchors has become the majority of the anchors. The population of network anchors from three provinces in the northeast of China is the largest, accounting for $15.03 \%$. The industry of live streaming in 2017 returns gradually to a reasonable level under the rigid supervision, the pattern of giants has shaped, and the reshuffle has begun. By the end of June 2017, there were about 270 live streaming platforms in online operation, with 41 platforms new and 6 platforms closed. As shown in Table 1 "Distribution of Anchor Platforms of TOP 50 Chinese Network Anchors in the First Half of 2017”: Huya, DouYu, Panda TV are the main force, the pattern of giants is obvious and the game platforms have outstanding advantages.

Table 1 "Distribution of Anchor Platforms of TOP 50 Chinese Network Anchors in the First Half of 2017”

\begin{tabular}{|l|l|}
\hline Live streaming platform & $\begin{array}{l}\text { Distribution of Anchor Platforms of TOP } 50 \\
\text { Chinese Network Anchors }\end{array}$ \\
\hline Huya & 16 \\
\hline DouYu & 15 \\
\hline Panda TV & 11 \\
\hline Longzhu & 4 \\
\hline Quanmin Live & 2 \\
\hline Zhanqi TV & 2 \\
\hline
\end{tabular}

(Data sources: Analysis Report of the Development of Chinese Live Streaming Industry in the First Half of 2017)

Let's see the current situation of college student anchors. According to data of our research team, the younger trend of network anchors is very obvious, and college student network anchor is the main group of Chinese network anchors.

According to the data, Huajiao launched the first "Campus Channel", with millions of campus anchors, covering $98.7 \%$ of the whole country, which has promoted the more rapid development of college student network anchor industry. These college student anchors are mainly from colleges and universities across the country, and the form of live streaming, affected by majors, is diversified. The proportion of students in media and art major as network anchor is the largest and the number of female college student anchors is the largest. These college student network anchors perform various talent shows according to their professional specialty, such as: singing, dancing, chatting, 
conducting advertising, education, sports, games and etc. Recently, more students from elite universities joined the network anchors with their high academic qualifications and high intelligence quotient to exert their specialties. Through the "live streaming + education" mode, they spread their cultural knowledge to realize their personal values and obtain high incomes, at the same time, will also gradually change the vulgar phenomenon of live streaming and achieve the network self-purification.

\section{The development plight of college student network anchors}

Driven by hardware upgrades such as smart phones and broadband speed and the influx of capital into the live streaming market, the live streaming has ushered in a popularization era where everyone can be an anchor. However, behind the prosperity of live streaming market, the quality of college student network anchors is different, and they show their special prowess to attract fans. Chaotic phenomena such as pornography, violence, routine, and data fraud occur frequently. The scarce high-quality contents and excellent anchors become the target live streaming platforms compete for. This disorder, vulgar, and extreme development trend has put the live streaming industry into troubles. It is necessary to relive the industry pain points and achieve the integration of industrial resources to promote network live streaming to develop continuously and healthily.

\subsection{It is hard for college student network anchors to become "Internet Celebrity"}

Network live streaming platform is a universal platform for everyone, and each of us can be an anchor. For example, many students from Tsinghua University and Peking University are in the Huajiao as tutor anchors. The number of students from School of Media and Art as anchors exceeds 25\%. Beautiful anchor show, sports and making friends, the barrage exchanges of TV series, punster anchor, teacher anchor, baking live show, mechanic show, and almost half of Internet users are involved in live streaming, some are on impulse, and some for making a living. Because of the popularization of live streaming and the high degree of participation, it is hard for college student network anchors to become Internet celebrity.

Affected by live streaming contents, broadband, national policies, aesthetic fatigue, academic pressure of college students, time limit and so on, the fans attracted of live streaming can hardly be sustained for a long time. Fixed live streaming platform, floating college student network anchors. Most college student network anchors are updated soon, and the churn rate of college student network anchors is as high as $25 \%$. For instance, mobile games, because of the fanaticism of fans and low-cost production, attracted so many teams, but also the problems such as cheating ranking, plagiarism, and copycatting. But after the fanaticism, the recognition of competitive products wins, simple and rough production exiting from the market, and low-level game anchor also quitting the game platform. The live streaming contents of some college student network anchors violate the bottom line for the attention. When all kinds of live streaming chaos appear one after another, more vulgar contents are produced, leading to the negative impression of the public on the industry of college student network anchors. Someone attributed the popularity of the college student network anchors industry to the hormone economy. How long can the hormone economy with scandals of college student network anchors such as ambiguity, pornography, and condom-selling last?

\subsection{The scarcity of high-quality contents and excellent anchors}

In pursuit of the hormone economy, the vulgar contents such as making love, spicy jokes, and nude shows flock. The contents of each platform is about the same, either pornography or violence, game, making friends, singing, and dancing, with little high-quality contents. Shallow and unitary contents cannot meet the needs of users in the face of high operating costs. The rapid development of the Internet era urgently needs the live streaming platforms to innovate in contents and forms.

Due to the low requirements of live streaming, the exposure of college student network anchors increases gradually, and the quality of anchors is different. There is relatively fewer excellent anchors, and college student network anchors who are uneducated and take low-level means such as vulgar language, pornography and violence to attract fans are more and more. Problems of vulgar 
contents, homogeneous competition, data fraud, policy tightening, and broadband limit have put the industry of college student network anchors in troubles.

\subsection{High pressure of college student anchors}

According to data of research team, college student network anchors are not what the Internet describes to earn ten thousand yuan a month easily with low cost and high income, however, most college student anchors are under lots of pressure.

First of all, live streaming needs to take up long time and anchors need to stay up late. Not all most college students are good at the live streaming, which requires lots of preparation work and long live time, and it is difficult to attract fans. Some college students conduct live streaming at 22:00 when fans are most active till the next morning to strengthen the attraction of fans. It probably brings high income, however, it is not a long-term solution. As for college student, on one hand, the academic pressure is heavy and on the other hand, it is hard for them to keep staying up late in the live streaming. This live streaming also has severely affected regular schedule and life and learning habits of college student.

Secondly, anchors need to go outside for live streaming. In order to win more fans, some college student anchors go outside for live streaming at 3:00 am. For college students, especially female students, the schedule is unreasonable, also it is filled with many unknown risk factors, so it is difficult for many college student anchors to persevere with live streaming.

Thirdly, there is malicious and vulgar harassment from fans. Anchors need strong psychological quality because the quality of the audience is different. In the face of malicious and vulgar fans, anchors need to become tolerant, defuse tensions or awkwardness with wisdom, or the live streaming cannot to go on.

\section{Development strategy of college student network anchors}

With today's rapid development of ever-changing Internet, there are often situations where the issuing of national policies and regulations lags behind the development of emerging industries. However, targeted laws and regulations will gradually play its role in the running-in with the market. The same is true of the blowout expansion of live streaming industry. The effective interaction between the four parties, "government+capital+platform+anchor" and the reshuffle in the industry forms the green and healthy pattern. Those shiftless live streaming platforms with low quality and college student network anchors will be eliminated.

\subsection{The supervision of government departments}

Everything has its good and bad sides, and the same is true of college student network anchors. As the emerging industry, it develops rapidly and causes lots of problems too. Nothing can be accomplished without norms or standards. In order to promote the continuous and prosperous development of college student network anchor industry, it is necessary to strengthen the constraints of laws and strict supervision of The Ministry of Public Security of the People's Republic of China, Office of the Central Leading Group for Cyberspace Affairs, Ministry of Culture of the People's Republic of China, Ministry of Industry and Information Technology of the People's Republic of China, and State Administration of Press, Publication, Radio, Film and Television of the People's Republic of China. On the morning of April 13, 2016, the Beijing Self-Discipline Convention on Network Live Streaming Industry stipulated that the anchor should carry out real-name authentication and juveniles under the age of 18 cannot register to be an anchor. From the end of July 2016 to the end of October 2016, The Ministry of Public Security of the People's Republic of China conducted a special rectification of network platform nationwide. In July 2016, the Ministry of Culture of the People's Republic of China issued the Notice on Strengthening the Management of Network Performance, regulating the contents of online game anchor and network art performance. On September 12, 2016, the State Administration of Press, Publication, Radio, Film and Television of the People's Republic of China also issued the Notice on Issues Concerning the Management of Live Streaming Services of Network Audio-visual Programs, requiring the live streaming agencies 
of network audio-visual programs to work with certificates.

With the constant innovation of government network supervision technology and the establishment of supporting regulatory team, the regulatory mechanism will be more sound. For some unqualified college student network anchors, the network platform will face greater rectification. After the guidance and supervision of relevant government departments, the industry of college student network anchor is gradually taking the path of healthy development.

\subsection{To integrate capital market and expand functions of live streaming platforms}

With the further development of mobile live streaming technology, the public prefers the live streaming with strong interaction and promptness. In the future, live streaming will no longer just remain in the pan-entertainment industry of games, chatting and sports, it will permeate the entity industry and serve industry and other industries, and "live streaming+" will be the standard configuration for all business.

On the afternoon of September 4, 2017, Shunya International Brand Consulting(Beijing) Co., Ltd announced that it will acquire $48.25 \%$ of the stock equity of Inke's subject of operation with RMB2,895 million, becoming the largest shareholder of Inke. This means that after going through , capital access, market integration and the implementation of new policies in the first year of live streaming in 2016, the live streaming industry began to undergo super integration, and start the pace of the transformation and upgrading and rebuilding of the industry's ecology.

In order to develop live streaming platforms, innovation in contents and form is very important. High-quality contents is the core competitiveness, also the key to the survival of live streaming platform in the future. And the unitary profit model in the future can hardly support the high cost of platform operation, which requires the platform to find a new business model. With the acquisition and reorganization of homogeneous live streaming platforms, the contents of the live streaming in vertical field will become the trend. The vertical field generally comes with content advantages and loyal user groups. Live streaming in vertical field such as live streaming + E-commerce, live streaming + education, live streaming + finance, live streaming + sports, and live streaming + traveling are occupying the live streaming market, including socializing, advertising, professions and so on, with live streaming evolving into an industry, which shows its enormous energy.

\subsection{Self-improvement and innovation of college student network anchors}

As an indispensable part of live streaming, with the low requirements, low cost, free time, easy time-killing, rich content, extensive participation, and strong interaction, college student network anchors obtain higher returns easily and show the blowout growth. It is these college student network anchors with their own speciality that attract people groups with different needs, and it is the nature of popularization that offers the opportunity for fans to interact with the idol anchor, and even to become the idol of the platform by various means, therefore, college student network anchors obtain the recognition of fans in the wave of technology revolution of new media. However, with the improvement of social cognition and attention, as well as the participation of real professional stars, there is an increasingly fierce competition for college student network anchors. Anchors with weak quality, capacity and characteristics gradually exit from the market. The high quality of college student network anchors has become the industry trend.

College student network anchors, if want to develop constantly in the industry, must make self-improvement and make innovations in form and content. The times of vulgar entertainment anchors will pass, an anchor with cultivation, culture, professionalism, innovative capacity will become the target live streaming platforms compete for. College student network anchors should systematically study the professional knowledge and conduct training on professional skills, and participate in the platform construction by means of shares so as to realize the effective allocation of superior resources.

\subsection{To actively guide college student network anchors to establish healthy values of live streaming}

Live streaming has moved deep into the life of each college student, whether becoming the 
anchor or watching live streaming has a profound impact on the outlook on life ad values of college students. To cultivate college students to have correct outlook on life and values is an important part of ideological and political education in colleges. The behaviors and contents of live streaming of college students should be included as part of the ideological and political education so as to guide them to establish correct concept of live streaming and healthy behaviors of live streaming. On the one hand, we can hold seminar about the problems of live streaming in the classroom to discuss the contents and form of live streaming in depth, and college students can realize what is correct and what is wrong in the discussion. On the other hand, we can promote correct and healthy attitudes and concepts of live streaming by means of new media, encourage outstanding college students to join college student network anchors to pass on cultural knowledge and spread positive energy to the society, to achieve personal value and to serve the society. In addition, we can combine the live streaming platform to realize the cooperation between school and enterprise and carry out the training on pre-empolyment professional quality and humane quality for college student network anchors, to create employment opportunities for college students, regulate the live streaming behaviors of college students, and purify the space of live streaming.

\section{Acknowledgements}

This paper is the partial achievements of the program "Empirical Study on the Impact of Network Anchor Subculture on the Core Values of College Students" (17JK1044) of Education Department of Shaanxi in 2017.

\section{References}

[1] Development Current Situation and Market Trend Prediction of China Network Live Streaming Market of 2017, Source: ChinaIRN.COM, 2016.12

[2] Wang Yichen, Capital Access, Market Integration and the Implementation of New Policies Live Streaming in Period of Stable Growth, Source: Economic Daily, 2017.3.30

[3] Analysis Report of the Development of Chinese Live Streaming Industry in the First Half of 2017, Source: Economic Information Daily, 2017.8.24

[4] Super Integration in Live Streaming, the Trend of Extension of Industry Chain, Source: Xinhua News Agency, Editor: Wang Bingkun, 2017.9.5

[5] Chen Bo, From Live Streaming Life to Scene Innovation: The Plight and Solution of Online Video Live Streaming Platforms, Broadcasting Realm, 2016.11

[6] Pan Di, Calm Thinking on the Hit of College Student Network Anchors [J].Today's Massmedia, 2016.2 